\title{
Biological Consequences of Psychological Distress in Caregivers of Children with Autism Spectrum Disorder and its Potential Relevance to Other Chronic Diseases Including Cancer
}

\author{
L. Dijkstra-de Neijs ${ }^{1}$ • P. J. M. Leenen ${ }^{2}$ - J. P. Hays ${ }^{3}$ • E. S. van der Valk ${ }^{4}$ R. Kraaij ${ }^{5}$ • E. F. C. van Rossum ${ }^{4}$. \\ W. A. Ester ${ }^{1,6,7}$ (iD
}

Published online: 4 July 2020

(C) The Author(s) 2020

\begin{abstract}
Purpose of Review Caregivers of children with a chronic illness are a neglected group in medical research and patient care, and are frequently confronted with chronic psychological distress. The biological consequences of this chronic distress are unclear but highly relevant, as these caregivers have a lifelong task in caring for their child. In this review, the authors specifically describe caregiver distress related to autism spectrum disorder (ASD), but the review may be relevant to other chronic diseases, including cancer.

Recent Findings Epidemiological evidence illustrates the increased mortality risk in caregivers of children with ASD although some individual factors appear to diminish these risks. Biological studies demonstrate that caregiver distress can lead to dysregulation of the hypothalamic-pituitary-adrenal-axis, a pro-inflammatory state of the immune and central nervous system, and gut microbiome imbalance.

Summary Caregivers of children with a chronic illness like ASD deserve more health-related attention with respect to their psychological and physical well-being. Such attention would benefit individual caregivers, as well as their children, as both are highly interconnected. Structural psychological and physical screening of caregivers can be considered.
\end{abstract}

Keywords Autism spectrum disorders · Caregiver distress · Endocrine system of cortisol $\cdot$ Immune system $\cdot$ Microbiome

\section{Introduction}

Distress is defined as a feeling of extreme worry, sadness, or pain [1], which can be the result of chronic and/or overwhelming stress. Stress is a biological and psychological adaptation to demanding circumstances, or the anticipation thereof. Chronic and/or overwhelming stress may shift from a

This article is part of the Topical Collection on Cancer Epidemiology

W. A. Ester

w.ester@youz.nl

1 Sarr Expert Centre for Autism, Youz Child and Adolescent Psychiatry, Dynamostraat 18, 3083 AK Rotterdam, The Netherlands

2 Department of Immunology, Erasmus MC, University Medical Center Rotterdam, Dr. Molewaterplein 40, 3015 GD Rotterdam, The Netherlands

3 Department of Medical Microbiology \& Infectious Diseases, Erasmus MC, University Medical Center Rotterdam, Dr. Molewaterplein 40, 3015 GD Rotterdam, The Netherlands balanced mental and physical equilibrium, in which stress has an evolutionary benefit of adaptation, toward distress. Caring for a child with a chronic illness (including a child with autism spectrum disorder or ASD) generates an increased burden of stress on caregivers [2••] which can lead to caregiver distress. A certain amount of stress is common in all caregivers [2••], originating from everyday tasks of caring for a

4 Department of Internal Medicine, Division of Endocrinology, Erasmus MC, University Medical Center Rotterdam, Dr. Molewaterplein 40, 3015 GD Rotterdam, The Netherlands

5 Department of Internal Medicine, Erasmus MC, University Medical Center Rotterdam, Dr. Molewaterplein 40, 3015 GD Rotterdam, The Netherlands

6 Parnassia Psychiatric Institute, Kiwistraat 43, 2552 DH The Hague, The Netherlands

7 Department of Child and Adolescent Psychiatry, Curium-LUMC, Leiden University, Endegeesterstraatweg 27, 2342 AK Oegstgeest, The Netherlands 
family member, in most cases a child [2••]. Caregiver distress may be reflected in increased psychological and/or socioeconomic problems, as well as physical symptoms such as an increased frequency of cardiometabolic and oncologic disease, an imbalanced immune system, and potential changes in the microbiome of caregivers. Specifically, caregivers of children with ASD show a higher incidence of cancer and vascular disease morbidity and mortality compared with caregivers of typically developing children [3・•]. Yet best practice guidelines for children with ASD do not include the well-being of their caregiver.

The effect of caregiver stress, possibly leading to distress, on the psychological health of caregivers has been widely reported. The caregivers of people with a variety of physical and psychological conditions show an increased prevalence of anxiety and depression, with caregivers twice as likely to make use of mental healthcare facilities compared with noncaregivers [4•]. They also experience more physical discomfort such as headaches, backaches, muscle soreness, and fatigue [5]. Additionally, when taking care of a child with a lifelong or life-threatening illness, such as cancer, caregiver distress increases due to the everyday uncertainty of the course of the condition and the persistent negative consequences of the disease to the child's life [6]. If a child presents with severe emotional or behavioral problems, then caregiver distress may also be increased. Caregivers of children with a psychiatric condition also experience increased marital problems [7, 8]. Further, additional financial costs, or loss of finances by having to cut back on working hours, or even having to quit a job, can all contribute to caregiver distress [4•]. Unfortunately, in such cases, there is a higher probability that the mental healthcare needs of the caregiver will be unmet [4•]. Worryingly, such caregiver distress may increase problematic behavior in the child with ASD, possibly leading to more severe behavioral problems within ASD children, creating a vicious circle of increasing distress and behavioral problems, which is hard to break $[9,10]$.

Therefore, the current review focuses on the effect of stress/ distress on the brain-body interaction of caregivers of children with ASD. Specifically, we aim to provide a holistic overview of the physical consequences. We focus on caregivers of children with ASD as studies show that these caregivers experience higher levels of psychological caregiver distress than caregivers of a typically developing child or a child with other serious medical conditions such as asthma [11] or Down syndrome [2••]. Caregiver distress in ASD is increased due to several factors, including the notion that ASD is not a physically visible condition (in contrast to Down syndrome for example) $[2 \bullet \bullet$. Additional factors which add to caregiver distress are as follows: the expected life outcomes of children with an ASD diagnosis, the experienced societal stigma due to unfamiliarity with ASD, and a high prevalence of comorbidities (which can be up to $70 \%$ in children with ASD [2]). Further, the early onset of ASD, with accompanying dysregulation and/or social engagement problems, is frequently already present when the child is just a few months old [12]. This can lead to an early onset of caregiver distress in often young caregivers and may be combined with a grieving process when expectations regarding parenthood turn out differently.

Most outcomes within caregiver distress studies are based on self-reports, presenting perceived parental distress. However, self-report questionnaires are sensitive to bias [13••] and do not always correlate well with physiological stress markers (salivary cortisol, salivary alpha-amylase, and blood pressure) [14]. Although the correlation between perceived and biological stress is marginally substantiated, both are known for their contribution to a variety of physical conditions, such as cardiovascular disease [15]. In this integrated review, we critically evaluate recent literature from the past 5 years or earlier relevant papers focussing on new findings on caregiver distress from a biological point of view, and specifically caregivers of children with ASD. Literature searches were performed in November and December 2019 using PubMed and Google Scholar. Given the limited literature available on the subject, also examples from closely related caregiving fields are provided. We describe the biological consequences of caregiver distress from the cardiometabolic, endocrine, immunological, and microbiota perspective. In this respect, we hope to raise awareness of the effect of stress/ distress in caregivers of children with ASD, while also providing integrated information that may be useful for future research into the caregivers of children and adults with other chronic illnesses.

\section{Caregiver Distress: Cardiometabolic Consequences}

\section{Individual factors in the caregiver-care recipient dyad determine mortality risk.}

The higher stress burden in caregivers of children with ASD is reflected in a significantly increased risk of mortality, as observed in a study among mothers of children with ASD, whose mortality due to cancer was found to be approximately $50 \%$ higher compared with mothers of typically developing children [3••]. This is striking, as for caregiving in general results tend to be different. Whereas some earlier studies found that caregiving in general is associated with an increase in mortality risk of up to $63 \%[16,17]$, more recent studies found a protective effect of caregiving, with hazard ratios as low as 0.72 for caregivers versus non-caregivers $[18,19]$. This leads to the hypothesis that it is probably not caregiving per se that leads to increased mortality, but that there are distinct individual factors in the caregiver-care recipient dyad that determine an individual's susceptibility to increased mortality. 
Importantly, the amount of stress experienced by caregivers has been found to be an important factor in the risk of their mortality [20].

\section{Stress Contributes to Cardiovascular Diseases}

The concept of "stress", and its influence on cardiovascular diseases, has been studied from both a psychological and biological perspective. In the general population, both are known to contribute to cardiovascular morbidity and mortality. In one large case-control study involving over 25,000 cases and controls with or without myocardial infarction, the odds ratio for self-reported stress for having a myocardial infarction was in the same range as the odds ratio for classical risk factors such as hypertension, obesity, and smoking [15]. Moreover, when specifically focusing on the risk of stress in the home environment, the odds ratio for permanent stress at home (2.12) was among the highest contributors to the risk of myocardial infarction [15].

\section{Physiological Markers of Stress and Cardiovascular Diseases-the HPA-Axis}

With regard to physiological markers of stress, numerous studies have related dysregulation of the HPA-axis (which produces the stress-hormone cortisol) to adverse cardiometabolic outcomes. For example, cortisol is a strong influencer of several cardiovascular parameters. It affects adipose tissue and induces insulin resistance, hypertension, and dyslipidemia [21]. In cases of extreme exposure to cortisol, such as Cushing's syndrome, patients develop severe abdominal obesity, hypertension, type 2 diabetes, and if left untreated, will rapidly die from vascular complications [22]. However, it is believed that not only exposures to extremely high amounts of cortisol predispose to cardiovascular diseases but subtle disturbances of the HPA-axis may also affect cardiometabolic health [23]. Although inconsistencies exist in the literature, there is a general pattern indicating that greater responsivity of the HPA-axis, reflected in morning awakening cortisol levels and acute stress reactivity, is associated with increased carriage of abdominal fat [24]. Moreover, when considering long-term cortisol levels, measured in scalp hair, additional associations have been found, such as the relationship between higher cortisol levels and abdominal obesity [25] or cardiovascular diseases [26].

\section{The HPA-Axis in Caregivers of Children with Autism Spectrum Disorder}

Studies evaluating the HPA-axis in caregivers of children with ASD have been summarized recently in a systematic review [13••]. Most investigations determined saliva cortisol or assessed the cortisol awakening response or the diurnal rhythm. Together, these studies suggest a blunted cortisol awakening response and lower cortisol levels, demonstrating that giving care to a child with ASD affects the HPA-axis functioning of the caregiver. However, as caregiving represents a long-term duty, and cardiovascular events are induced by long-term exposure to glucocorticoids, it remains questionable whether the measurements used in these studies, which actually indicate short-term HPA-axis responsivity, are appropriate for studying the long-term consequences of caregiving. It is possible that measuring indicators of long-term cortisol levels, such as hair cortisol, can further elucidate this subject, as such measurements are associated with chronic stress [27, $28]$. In the field of caregiving, one study specifically assessed long-term cortisol levels of caregivers for individuals with ASD [29]. The authors found that caregivers who were selected on having a relatively high perceived stress score had lower long-term cortisol levels than less stressed controls. This is in line with the findings from short-term cortisol measurements, pointing to a general pattern of decreased HPA-axis activity in caretakers of children with ASD.

Conversely, another study that assessed long-term cortisol levels of caregivers of individuals with dementia found strongly increased cortisol levels, which even increased with the amount of stress that they perceived [30]. This may be due to the differences in taking care of a person with dementia versus a child with ASD. However, it could also be a consequence of the duration of caregiving, which was on average 40 months for the dementia caregivers but likely much longer for the ASD caretakers. Also, the age of the caregivers should be taken into account, which is relatively young for ASD caregivers. Moreover, in ASD caregivers, the cortisol levels decreased even further after 2 years, which may indicate that prolonged exposure to stress blunts the HPA-axis even further. Previously, it has been shown in post-traumatic stress and anxiety disorders that long-term exposure to stressful stimuli may actually desensitize the HPA-axis, decreasing its activity [31].

\section{An individual's Sensitivity to Cortisol}

With regard to the biological effect of cortisol, not only the level of cortisol should be considered but also the individual biological sensitivity. Importantly, differences in glucocorticoid sensitivity are associated to physical well-being [32] and correlate with cardiometabolic health. Specifically, individual genetic polymorphisms have been found that affect sensitivity to cortisol, and are associated with body composition, such as adiposity or insulin resistance [23]. Sensitivity to cortisol can also be subject to change over time. It is possible that a change in glucocorticoid sensitivity due to the stress of caregiving underlies the increased mortality risk for caregivers with children with ASD. In comparison, in the 12 months following the diagnosis of a child with cancer, their mother's biological 
sensitivity to cortisol gradually declined. This decline correlated to the increase in stress they experienced [33]. Therefore, it is possible that caregiving for individuals with ASD also affects glucocorticoid sensitivity.

In summary, although evidence is limited, giving care to children with ASD has been associated with increased mortality. The HPA-axis may be involved, generally showing reduced cortisol levels after long-term caregiving for children with ASD. As a decrease in cortisol seems contradictory to the increased cardiovascular risk, more research is needed, combining long-term cortisol measurements with assessments of glucocorticoid sensitivity at the cellular level and throughout the course of disease. Importantly, alterations in the set point of the HPA-axis can not only influence health status via the direct metabolic effects of cortisol but also indirectly via effects on the immune system. We will discuss the immunological perspective in the next paragraph.

\section{Caregiver Distress from an Integrated Immunological Perspective}

\section{Mental stress is reflected in an elevated inflammatory state and disturbed glucocorticoid response in peripheral blood leukocytes}

In recent years, it has become increasingly clear that human mental well-being is reflected in the inflammatory status of peripheral blood leukocytes [34•]. Even the driving forces of positive mood, i.e., pleasure-seeking "hedonic" versus prosocial "eudemonic" well-being, translate into different leukocyte inflammatory gene expression profiles [35]. Although counterintuitive, an increased inflammatory set point of leukocytes may imply a state of reduced immune responsiveness toward infection or immunization, as observed in aging or obesity [36]. In leukocytes from individuals exposed to prolonged adverse conditions, such as chronic stress, a common gene expression signature, indicated as "conserved transcriptional response to adversity" (CTRA), is observed. This involves elevated pro-inflammatory signaling, via transcription factors NF-kappaB and AP-1, leading to increased production of pro-inflammatory cytokines including IL-6, TNF, and IL-1 [37]. A further characteristic of CTRA is decreased interferon type I signaling, which probably contributes to reduced responsiveness to viral infections [37]. This phenotype is carried especially by monocytes in circulation and related macrophages and dendritic cells in tissue. The CTRA profile is associated with increased risk of cardiovascular disease and cancer [38]. Sympathetic nerve signaling drives stress-related myelopoiesis in the bone marrow and development of monocytes with a CTRA profile, as has been shown in human studies as well as animal models [39॰•]. This adrenergic neuronal signaling mediates the fight-or-flight stress response in general and stimulates differentiation of monocytes that appear to be increasingly glucocorticoid resistant. Probably, this counterbalances the effect of generally increased cortisol levels in chronically stressed individuals [27] and contributes to their monocyte pro-inflammatory profile, as glucocorticoids are major inhibitors of inflammation [32].

Animal models of chronic stress, such as repeated social defeat models, have shed light on the interactions between the central nervous system (CNS) and the periphery. These models have shown that the resident macrophages of the CNS, the microglia, are activated under circumstances of elevated stress to produce pro-inflammatory cytokines and chemokines [40]. These mediators, in turn, activate endothelial cells to recruit circulating monocytes into the CNS. Infiltrating monocytes probably contribute to the CNS proinflammatory state due to their relative GC insensitivity.

Significant differences exist between individuals regarding their perceived levels of stress, the consequences for mental well-being, and subsequent reflection into leukocyte inflammatory profile. The underlying parameters contributing to susceptibility and resilience are multifactorial and include gender as well as inflammatory responsiveness [41, 42]. In the latter, individual differences in cortisol sensitivity are important, as indicated above. Moreover, results from animal models using wild-type and gene-knockout mice suggest that IL-6 plays a critical role in the interindividual differences in stress susceptibility $[43 \cdot, 44]$.

\section{Immune Consequences of Stress Induced by Caregiving to Children with ASD}

Specific information concerning the immune effects of distress in caregivers to children with ASD is fragmentary but matches findings in other caregiver stress-related conditions [45-47]. Caregiver distress in general leads to disturbances of the HPA axis and increased glucocorticoid resistance, as indicated above. This relates to decreased dampening of inflammatory responses, which explains the direct positive association between the levels of distress and inflammatory parameters as observed in caregivers of individuals with cancer [33, 48]. The inflammatory profile of caregivers of children with ASD is unknown, but interestingly their leukocytes show a lower mitochondrial health index compared with controls, which is associated with higher perceived stress and lower positive mood [49]. This finding is in line with the notion that monocyte pro-inflammatory activation is linked to suppression of mitochondrial function [50].

In immune effector function, caregiver distress is reflected in decreased levels of secretory IgA, suggesting reduced immune barrier function at mucosal surfaces and diminished antibody responses to immunization $[51,52]$. These deviations are most evident in older caregivers, but in younger individuals, this condition also leads to increased immune 
aging as indicated by advanced $\mathrm{T}$ cell differentiation. Although steady-state neutrophil anti-bacterial function appears not significantly different between caregivers and controls, those with higher psychological morbidity have poorer neutrophil phagocytosis capacity, suggesting decreased function of the cells under challenging conditions [53].

An interesting notion surfacing in recent years is that altered immune status upon stress exposure correlates with altered composition of the microbiota, in particular, in the gut [54]. Importantly, the interaction between microbiota and the intestinal immune system appears to be bidirectional, indicative of their mutual interdependence [55].

\section{Caregiver Distress: Microbiota}

The study of the human microbiota and its influence on the health of individuals has been a "hot topic" of research since the general availability of DNA sequencing methods more than a decade ago. The gut microbiota comprises microorganisms (mainly bacteria, but also fungi and viruses) that reside in the lumen and wall of the gastrointestinal tract. A number of genes encoded by the microbiota are estimated to exceed one million, whereas humans only express approximately twenty thousand genes. A very large number of association studies have now linked the gut microbiota to many different diseases and traits. The validity of these association studies is now being explored in a wide range of animal models, large human population-based cohort studies, large human case-control studies, and large human intervention studies. However, despite these ongoing efforts, it is only relatively recently that scientists have begun to study and appreciate the potential effect of the human microbiota on mental health and disease [56•], [57]. It is therefore not surprising that the majority of microbiota work currently published has focused on the association between microbiota profiles and mental diseases within patients themselves, with very few data available regarding the role of the human microbiota in the mental and physical health of caregivers. In fact, currently we find no studies that have been performed to investigate the influence of the human microbiota in caregivers of ASD dependents, although many data relate the human microbiota to ASD per se (for a review see Li et al. [58]). However, as previously mentioned, it is known that the mental health of individuals may be associated with variation in the human gut microbiota [59] and that mental health issues such as stress and depression affect caregivers of children with ASD more than caregivers of non-autistic children [60]. Therefore, understanding the interactions between the gut microbiota and human host may provide a platform upon which future interventional studies into caregiver distress can be based.

The mechanisms by which the human microbiota and specifically the gut microbiota could influence mental health include microorganism interactions with the central nervous system (autonomic, neuroendocrine, and enteric systems), the immune system, and interactions of metabolites produced in the gut that reach the brain through the circulatory system. The subsequent mental health pathologies induced include nonhomeostatic disturbances in neurotransmitter balance, increased chronic inflammation, and/or increased HPA-axis activity [61•]. Additionally, stress and depression may be factors leading to the preferred growth of bacteria that encourage gut microbial imbalance, malabsorption, or regional formation of increased intestinal permeability [62]. This leads to the concept of "reverse causation," i.e., the influence of behavior on the composition of the gut microbiota, a concept that should not be neglected when studying distress and microbiota interactions in caregivers of children with ASD.

Evidence suggesting that the gut microbiota plays a role in mediating bidirectional communication between the gut and the brain is accumulating [63, 64]. Most studies on the microbiota-gut-brain axis have been performed in animal models, particularly in germ-free mice. These models have led to fundamental insights into the importance of gut bacteria for neurodevelopment and behavior. Germ-free mice differ from conventional mice over a range of functional behavioral classes, such as learning, memory, social interactions, stress response, and anxietyand depressive-like symptoms [65]. Further, several animal studies provide evidence that the gut microbiota has an impact on the neurobiological features of depression [66-72], with microbiotal transplantation from either stressed or obese animals to control animals showing significant alteration in anxiety-like behaviors [73]. Only a few studies have addressed the relationship between gut microbiota and depression in humans [74]. A larger, population-based study recently reported depletion of butyrate-producing bacteria Coprococcus and Dialister in individuals with depression [56•]. Such large-scale metagenomic studies (addressing both bacterial species composition and their collective functions) are now guiding the field toward a mechanistic interpretation of the gut-microbiota-brain axis. This in turn will deliver more detail on the role of the microbiota in caregiver distress.

\section{Holistic Overview of Endocrine, Immune, and Microbiota Interactions in Caregiver Distress}

In Fig. 1, we have attempted to integrate recent findings concerning the consequences of distress on endocrine, immune, and microbiota systems in caregivers of children with ASD. The figure is based on findings in this specific population and on stress-induced physical outcomes in general. In brief, stress activates neuronal pathways as well as related 
Fig. 1 Holistic view of endocrine, immune, and microbiota alterations in caregiver distress. Abbreviations: $A C T H$ : adrenocorticotropic hormone; CTRA: conserved transcriptional response to adversity; $D C$ : dendritic cell; $G C$ : glucocorticoid; $H P A$ : hypothalamus-pituitaryadrenal axis; $M f$ : macrophage; SNS: sympathetic nerve signaling (adrenergic)

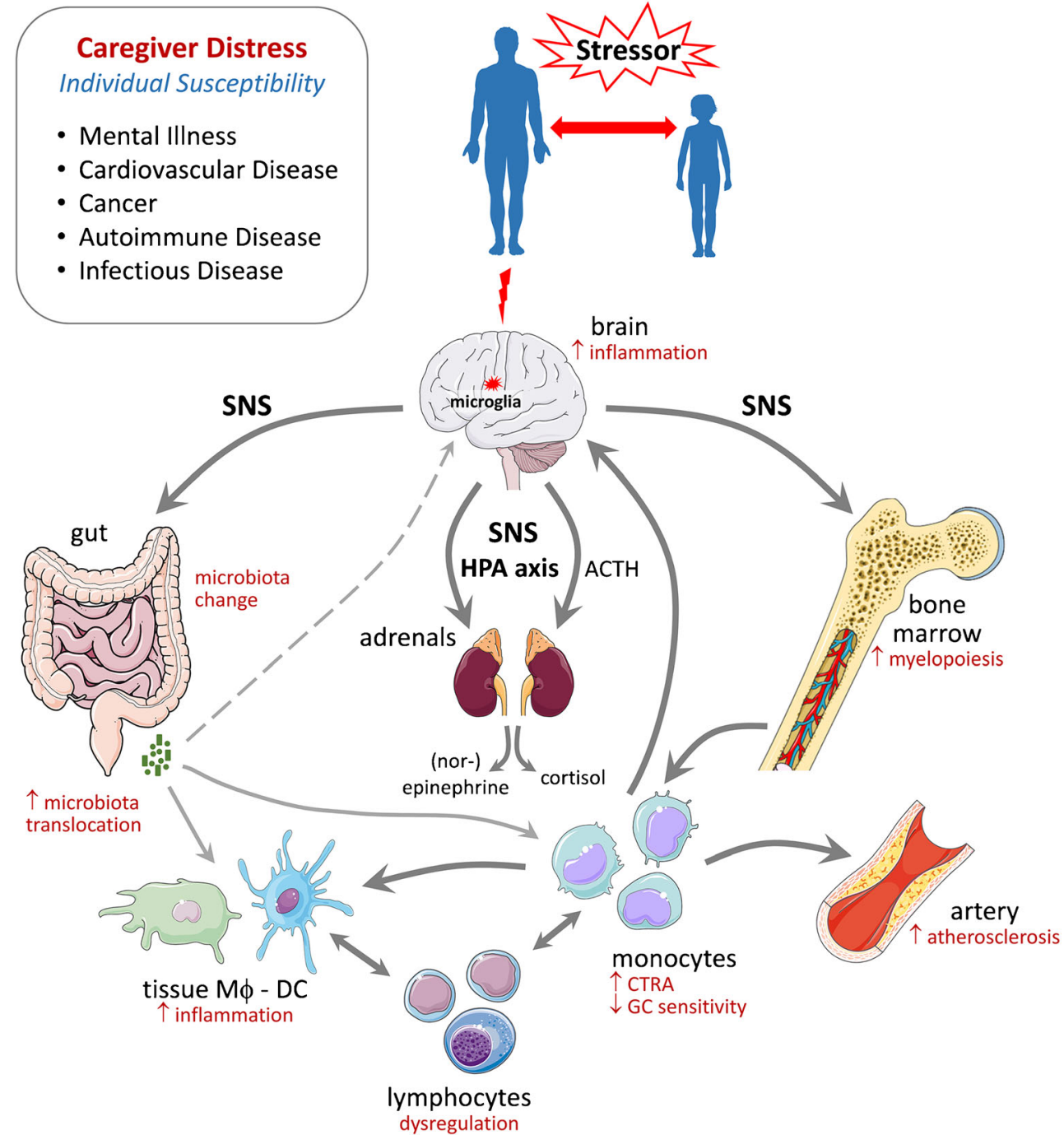

microglia in the brain, which assume an elevated inflammatory state. Sympathetic neuronal signaling (SNS) to the bone marrow, using norepinephrine as neurotransmitter, stimulates myelopoiesis. This drives generation of monocytes with a proinflammatory CTRA expression profile, indicated by increased expression of inflammatory cytokines like IL-1, TNF, and IL-6, and decreased glucocorticoid sensitivity. These monocytes migrate to the brain as well as to peripheral organs and differentiate into tissue macrophages and dendritic cells. Similar sympathetic signaling to the adrenal medulla leads to release of (nor-) epinephrine in the circulation, while activation of the HPA-axis liberates ACTH from the pituitary, stimulating cortisol production by the adrenal cortex. Further sympathetic signaling to the intestines, as well as decreased levels of secretory IgA, may lead to alterations in gut barrier function and increased translocation of microbiota into the systemic circulation, commonly indicated as the "leaky gut" $[75,76]$. Increased exposure to microbial components activates tissue macrophages, dendritic cells, and circulating monocytes in a pro-inflammatory manner. This may contribute to various pathogenic processes, including growth of arterial plaques leading to cardiovascular disease. Whether translocated microbiota also directly influence the brain is unknown, although crosstalk between mucosal tissue, immune cells, and gut microbiota probably underlie the changes observed in gut microbiota composition observed in caregivers.

The chronic presence of mental "stressors" is thought to have long-standing effects on future responses to stress. This imprinting refers not only to neuronal circuits but also to microglia and peripheral blood monocytes and related cells [77, 78]. For the latter myeloid cells, increased inflammatory responses related to prior exposure are known as "trained innate immunity." This is regulated by changes in the epigenetic status of pro- and antiinflammatory genes, which in turn is closely intertwined with the activity and balance of metabolic pathways, in particular, 
glycolysis and oxidative phosphorylation. Together, the chronically elevated inflammatory status of individuals exposed to adverse levels of stress and the imprinting occurring at neuronal as well as immunological level are likely explanations for the elevated prevalence of chronic conditions such as cardiovascular disease and cancer among caregivers of children with ASD.

Several studies point to biological parallels between the caregivers of individuals with ASD and other chronic illnesses, such as cancer. Specifically, mothers of children who were newly diagnosed with cancer displayed a decline in biological sensitivity to cortisol, which was correlated to increased distress [33]. Additionally, distress can lead to disturbances of the HPA axis and increased glucocorticoid resistance, which is related to a decreased dampening of inflammatory responses - as observed in caregivers of individuals with cancer [33, 48]. In addition, distress (and specifically depression) may be associated with the composition of the gut microbiota [56•] [75]. Thus, it seems likely that the endocrine and immunological consequences of psychological distress in ASD are equally relevant for caregivers of individuals with cancer.

\section{Possible Treatment Targets}

Although immunological interventions are scarce and aimed at decreasing the leukocyte inflammatory status in general, it is the manipulation of the gut microbiota that potentially offers novel therapies for caregivers experiencing chronic stress/distress. Potential interventions include dietary adaptations, use of pre- and probiotics or psychobiotics, and fecal microbial transplants. In this respect, psychobiotics is a term used to describe prebiotic mixtures of compounds that promote the growth of "beneficial" gut bacteria that positively influence mental health, while probiotic mixtures are live microorganisms that tend to promote gut, and hence mental health [79]. As just one example, Talbot et al. studied the effect of coordinated probiotic/prebiotic/phytobiotic supplementation (FundaMentals, Amare Global, Irvine, CA, USA) on 32 healthy individuals suffering from "moderate" levels of psychological stress. The authors found statistically significant improvements in both positive $(+25 \%$ Global Mood; $+44 \%$ Vigor) and negative $(-64 \%$ Fatigue; $-55 \%$ Depression; $-54 \%$ Anger; $-45 \%$ Tension; $-43 \%$ Confusion) mood state parameters in the supplement versus placebo group [80]. However, microbiota profiles vary between different individuals, meaning that further research relating these microbiota compositions to endocrine and immune responses is also required to generate an integrated overview of the mechanisms that stress and distress bring about in caregivers. Only with this, more effective treatments can be developed that specifically target the individual caregiver, and thus facilitate personalized medicine in this field.

\section{Implications}

Our exploratory review indicates that giving care to a child with ASD is associated with reduced mental health and disturbances of the endocrine system, the immune system, and the microbiota, altogether probably contributing to reduced general health status and increased mortality (including early cancer mortality), as observed within mothers of children with ASD [3]. Further, these implications could be broadly applicable to caregivers of individuals suffering from other chronic illnesses, such as cancer. Therefore, the inclusion of mental health screening for caregivers of children with chronic illnesses is advised. As the psychological and socio-economic consequences of caregiver distress are broad, a multi-layered screening would be appropriate. Psychological screening should not only include questions on caregiver distress but also on socioeconomic stressors, such as marital and financial problems and individual problems, minimally focusing on screening for anxiety and depression symptoms within the caregiver.

Preferably, the psychological screening for caregivers at risk for pathological consequences of caregiver distress can be supported by corresponding biomarkers, in which longterm cortisol levels combined with a cardiovascular risk profile might be suited. Although there is insufficient evidence to support strict recommendations for use in everyday clinical practice, we can conclude that the health of caregivers of children with chronic illnesses should not be overlooked. Possibly, primary healthcare physicians should consider additional screening for cardiovascular risk factors and cancer risks in caregivers of individuals with chronic illnesses.

To further elucidate this relationship, and specifically the influence of the HPA-axis, the assessment of long-term stress hormone levels and the determination of individual sensitivity to stress (both on a biological and psychosocial level) seem important targets for future research in this field. Also, new epidemiological cohort studies into the prevalence of various types of cancer in caregivers of individuals with chronic illnesses would create a better understanding of additional health risks in this population. The outcome of such research could be used to implement effective "good mental health education" practices, as well as the targeted screening of caregivers for increased psychological, cardiovascular, and cancer risks.

\section{Conclusions}

Chronic exposure to stress in caregivers of children with ASD (and other chronic diseases) may lead to an altered set point of immune and endocrine response systems that may be reflected in altered microbiota composition and gut-microbe interactions. Taken together, such changes mediate an elevated risk of cardiovascular disease, cancer, and autoimmune and infectious diseases in individual caregivers. However, significant 
individual differences exist in the vulnerability of caregivers to these changes, for example, individual stress responses differ substantially. Further, although it is exciting to consider that it might be possible to determine the individual risk of stress-induced disease on the basis of specific biophysical parameters, the exact identity of these critical parameters remains to be determined. Reducing mental stress in caregivers may be achieved by optimizing the caregivers' ability to cope with psychologically challenging conditions. Surprisingly, however, although studies evaluating distress levels in caregivers have been published since at least the 1990s, explicit physical and mental healthcare provision for caregivers remains scarce within everyday clinical practice. In this respect, it is tempting to speculate that nutritional and nutraceutical healthcare approaches - involving pre- and/or probioticsmight provide suitable complements to the current healthcare alternatives offered to caregivers.

Acknowledgments The authors would like to acknowledge Servier Medical Art for allowing the use of their graphical content in Fig. 1.

\section{Compliance with Ethical Standards}

Conflict of Interest The authors declare no conflicts of interest.

Human and Animal Rights and Informed Consent This article does not contain any studies with human or animal subjects performed by any of the authors.

Open Access This article is licensed under a Creative Commons Attribution 4.0 International License, which permits use, sharing, adaptation, distribution and reproduction in any medium or format, as long as you give appropriate credit to the original author(s) and the source, provide a link to the Creative Commons licence, and indicate if changes were made. The images or other third party material in this article are included in the article's Creative Commons licence, unless indicated otherwise in a credit line to the material. If material is not included in the article's Creative Commons licence and your intended use is not permitted by statutory regulation or exceeds the permitted use, you will need to obtain permission directly from the copyright holder. To view a copy of this licence, visit http://creativecommons.org/licenses/by/4.0/.

\section{References}

Papers of particular interest, published recently, have been highlighted as:

- Of importance

•. Of major importance

1. "DISTRESS | meaning in the Cambridge English Dictionary." [Online]. Available: https://dictionary.cambridge.org/dictionary/ english/distress. [Accessed: 10-Feb-2020].

2.• Hayes SA, Watson SL. The impact of parenting stress: A metaanalysis of studies comparing the experience of parenting stress in parents of children with and without autism spectrum disorder. J Autism Dev Disord. 2013;43(3):629-42 Systematic review with meta-analysis compairing 15 studies on caregiver distress in parents of ASD children $(n=638)$ with typically developing children $(n=896)$ and children with other disabillities $(n=825)$ such as Down syndrome, Cerebral palsy and intellectual disabillities. Experienced caregiver distress in parents of a child with ASD versus typicaly developing children resulted in a large effect size.

3.• Fairthorne J, Hammond G, Bourke J, Jacoby P, Leonard H. Early mortality and primary causes of death in mothers of children with intellectual disability or Autism spectrum disorder: A retrospective cohort study. PLoS One. 2014;9(12):1-15 Epidemiological study among mothers showing increased mortality when caring for a child with ASD compared to mothers of typically developing children.

4. Pilapil M, Coletti DJ, Rabey C, DeLaet D. Caring for the caregiver: supporting families of youth with special health care needs. Curr Probl Pediatr Adolesc Health Care. 2017;47(8):190-9 Review article describing caregiver distress as a multi dimensional concept including the fysical, emotional and financial toll of providing care.

5. Smith LE, Seltzer MM, Greenberg JS. Daily health symptoms of mothers of adolescents and adults with fragile $\mathrm{X}$ syndrome and mothers of adolescents and adults with autism spectrum disorder. J Autism Dev Disord. 2012;42(9):1836-46.

6. Bally JMG, Smith NR, Holtslander L, Duncan V, Hodgson-Viden $\mathrm{H}$, Mpofu C, et al. A metasynthesis: uncovering what is known about the experiences of families with children who have lifelimiting and life-threatening illnesses. J Pediatr Nurs. 2018;38(2018):88-98.

7. Seltzer MM, Greenberg JS, Floyd FJ, Pettee Y, Hong J. Life course impacts of parenting a child with a disability. Am J Ment Retard. 2001;106(3):265-86.

8. Hartley $\mathrm{S}$, et al. The relative risk and timing of divorce in families of children with an autism spectrum disorder. J Fam Psychol. 2010;24(4):449-57.

9. Smith LE, Greenberg JS, Mailick MR. The family context of autism spectrum disorders. Influence on the behavioral phenotype and quality of life. Child Adolesc Psychiatr Clin N Am. 2014;23(1): 143-55.

10. Zaidman-Zait A, et al. Examination of bidirectional relationships between parent stress and two types of problem behavior in children with autism spectrum disorder. J Autism Dev Disord. 2014;44(8): 1908-17.

11. Pinquart M. Parenting stress in caregivers of children with chronic physical condition - a meta-analysis. Stress Health. 2018;34(2) Wiley:197-207.

12. Charman T, Swettenham J, Baron-Cohen S, Cox A, Baird G, Drew A. Infants with autism: an investigation of empathy, pretend play, joint attention, and imitation: Dev. Psychol; 1997.

13.• Padden C, Concialdi-McGlynn C, Lydon S. Psychophysiological measures of stress in caregivers of individuals with autism spectrum disorder: a systematic review. Dev Neurorehabil. 2019;22(3):14963 Systematic review regarding Psychophysiological Measures of Stress in Caregivers of Individuals with Autism Spectrum Disorder, illustrating their HPA-axis disturbance.

14. Padden C, James JE. Stress among parents of children with and without autism spectrum disorder: a comparison involving physiological indicators and parent self-reports. J Dev Phys Disabil. 2017;29(4):567-86.

15. Rosengren A, Hawken S, Ônpuu S, Sliwa K, Zubaid M, Almahmeed WA, et al. Association of psychosocial risk factors with risk of acute myocardial infarction in 11119 cases and 13 648 controls from 52 countries (the INTERHEART study): casecontrol study. Lancet. Sep. 2004;364(9438):953-62.

16. Schulz R, Beach SR. Caregiving as a Risk Factor for Mortality The Caregiver Health Effects Study. JAMA. 1999;282(23):2215-9. 
17. Polenick CA, Leggett AN, Webster NJ, Han BH, Zarit SH, Piette JD. Multiple chronic conditions in spousal caregivers of older adults with functional disability: associations with caregiving difficulties and gains. J Gerontol B Psychol Sci Soc Sci. 2020;75(1): 160-72.

18. Leggett AN, Sonnega AJ, and Lohman MC, "Till death do us part: intersecting health and spousal dementia caregiving on caregiver mortality," J Aging Health, 2019.

19. O'Reilly D, Rosato M, Maguire A, Wright D. Caregiving reduces mortality risk for most caregivers: a census-based record linkage study. Int J Epidemiol. Dec. 2015;44(6):1959-69.

20. Fredman L, Cauley JA, Hochberg M, Ensrud KE, Doros G. Mortality associated with caregiving, general stress, and caregiving-related stress in elderly women: results of caregiverstudy of osteoporotic fractures. J Am Geriatr Soc. 2010;58(5): 937-43.

21. Fardet B, Fève L. Systemic glucocorticoid therapy: a review of its metabolic and cardiovascular adverse events. Drugs. 2014;74: 1731-17.

22. Nieman LK, Biller BMK, Findling JW, Newell-Price J, Savage MO, Stewart PM, et al. The diagnosis of Cushing's syndrome: an endocrine society clinical practice guideline. J Clin Endocrinol Metab. 2008;93(5):1526-40.

23. van der Valk ES, Savas M, van Rossum EFC. Stress and Obesity: Are There More Susceptible Individuals? Curr Obes Rep. 2018;7(2):193-203.

24. Incollingo Rodriguez AC, Epel ES, White ML, Standen EC, Seck1 JR, Tomiyama AJ. Hypothalamic-pituitary-adrenal axis dysregulation and cortisol activity in obesity: a systematic review. Psychoneuroendocrinology. 2015;62:301-18.

25. Noppe G, et al. Long-term glucocorticoid concentrations as a risk factor for childhood obesity and adverse body fat distribution. Int $\mathrm{J}$ Obes. 2016;40(10):1503-9.

26. Iob E, Steptoe A. Cardiovascular Disease and Hair Cortisol: a Novel Biomarker of Chronic Stress. Curr Cardiol Rep. 2019;21(10):116 Current Medicine Group LLC 1.

27. Stalder T, et al. Stress-related and basic determinants of hair cortisol in humans: A meta-analysis. Psychoneuroendocrinology. 2017;77: 261-74.

28. Kalliokoski O, Jellestad FK, Murison R. A systematic review of studies utilizing hair glucocorticoids as a measure of stress suggests the marker is more appropriate for quantifying short-term stressors. Sci Rep. 2019;9(1):11997.

29. Radin RM, Mason AE, Laudenslager ML, Epel ES. Maternal caregivers have confluence of altered cortisol, high reward-driven eating, and worse metabolic health. PLoS One. 2019;14(8):e0221354.

30. Stalder T, Tietze A, Steudte S, Alexander N, Dettenborn L, Kirschbaum C. Elevated hair cortisol levels in chronically stressed dementia caregivers. Psychoneuroendocrinology. 2014;47:26-30.

31. Staufenbiel SM, Penninx BWJH, Spijker AT, Elzinga BM, van Rossum EFC. Hair cortisol, stress exposure, and mental health in humans: a systematic review. Psychoneuroendocrinology. 2013;38(8):1220-35.

32. Quax RA, et al. Glucocorticoid sensitivity in health and disease. Nat Rev Endocrinol. 2013;9(11):670-86.

33. Walsh CP, Ewing LJ, Cleary JL, Vaisleib AD, Farrell CH, Wright AGC, et al. Development of glucocorticoid resistance over one year among mothers of children newly diagnosed with cancer. Brain Behav Immun. Mar. 2018;69:364-73.

34. Cole SW. The Conserved Transcriptional Response to Adversity. Curr Opin Behav Sci. 2019;28:31-7 Elsevier Ltd. This review provides an overview of recent studies showing the impact of chronic stress on the gene expression profile of circulating immune cells, myeloid cells in particular. Beta-adrenergic signaling to cells developing in the bone marrow mediates this alteration.
35. Fredrickson BL, Grewen KM, Coffey KA, Algoe SB, Firestine $\mathrm{AM}$, Arevalo JMG, et al. A functional genomic perspective on human well-being. Proc Natl Acad Sci U S A. 2013;110(33): 13684-9.

36. Frasca D, Blomberg BB, Paganelli R. Aging, obesity, and inflammatory age-related diseases. Front Immunol. 2017;8:1745.

37. Cohen S, Janicki-Deverts D, Doyle WJ, Miller GE, Frank E, Rabin BS, et al. Chronic stress, glucocorticoid receptor resistance, inflammation, and disease risk. Proc Natl Acad Sci U S A. 2012;109(16): 5995-9.

38. Simons RL, Lei MK, Beach SRH, Barr AB, Cutrona CE, Gibbons $\mathrm{FX}$, et al. An index of the ratio of inflammatory to antiviral cell types mediates the effects of social adversity and age on chronic illness. Soc Sci Med. 2017;185:158-65.

39.• Tawakol A, et al. Relation between resting amygdalar activity and cardiovascular events: a longitudinal and cohort study. Lancet. 2017;389(10071):834-45 This study demonstrates a direct association in humans between stress-related amygdalar activity, bone marrow response, and arterial inflammation, thereby providing a direct link between perceived stress, monocyte differentiation, and cardiovascular disease.

40. Weber MD, Godbout JP, Sheridan JF. Repeated Social Defeat, Neuroinflammation, and Behavior: Monocytes Carry the Signal. Neuropsychopharmacology. 2017;42(1) Nature Publishing Group: 46-61.

41. Finnell JE, Wood SK. Putative inflammatory sensitive mechanisms underlying risk or resilience to social stress. Front Behav Neurosci. 2018;12:240. Frontiers Media S.A.

42. Ménard C, Pfau ML, Hodes GE, Russo SJ. Immune and neuroendocrine mechanisms of stress vulnerability and resilience. Neuropsychopharmacology. 2017;42(1):62-80.

43. Nasca C, Menard C, Hodes G, Bigio B, Pena C, Lorsch Z, et al. Multidimensional predictors of susceptibility and resilience to social defeat stress. Biol Psychiatry. 2019;86(6):483-91 Using a mouse model the authors identify different predictors of stress susceptibility and resilience, including the in vitro inflammatory response of peripheral blood leukocytes to lipopolysaccharide.

44. Tsyglakova M, McDaniel D, Hodes GE. Immune mechanisms of stress susceptibility and resilience: Lessons from animal models. Front Neuroendocrinol. 2019;54:100771. Academic Press Inc.

45. De Andrés-García S, Moya-Albiol L, González-Bono E. Salivary cortisol and immunoglobulin a: responses to stress as predictors of health complaints reported by caregivers of offspring with autistic spectrum disorder. Horm Behav. 2012;62(4):464-74.

46. James Cy JE, Leader G. Parenting stress, salivary biomarkers, and ambulatory blood pressure: a comparison between mothers and fathers of children with autism spectrum disorders. J Autism Dev Disord. 2015;45(4):1084-95.

47. Lovell B, Moss M, Wetherell M. The psychosocial, endocrine and immune consequences of caring for a child with autism or ADHD. Psychoneuroendocrinology. 2012;37(4):534-42.

48. Sherwood PR, Price TJ, Weimer J, Ren D, Donovan HS, Given $\mathrm{CW}$, et al. Neuro-oncology family caregivers are at risk for systemic inflammation. J Neuro-Oncol. 2016;128(1):109-18.

49. Picard M, Prather AA, Puterman E, Cuillerier A, Coccia M, Aschbacher K, et al. A mitochondrial health index sensitive to mood and caregiving stress. Biol Psychiatry. Jul. 2018;84(1):9-17.

50. Chen Y, Zhou Z, Min W. Mitochondria, oxidative stress and innate immunity. Front Physiol. 2018;9:1487, no. OCT. Frontiers Media S.A.

51. Romero-Martínez Á, Moya-Albiol L. Stress-induced endocrine and immune dysfunctions in caregivers of people with eating disorders. Int J Environ Res Public Health. 2017;14(12):1560.

52. Whittaker AC, Gallagher S. Caregiving alters immunity and stress hormones: a review of recent research. Curr Opin Behav Sci. 2019;28. Elsevier Ltd:93-7. 
53. Vitlic A, Lord JM, Taylor AE, Arlt W, Bartlett DB, Rossi A, et al. Neutrophil function in young and old caregivers. Br J Health Psychol. 2016;21(1):173-89.

54. Hollins SL, Hodgson DM. Stress, microbiota, and immunity. Curr Opin Behav Sci. 2019;28. Elsevier Ltd:66-71.

55. Chen F, Stappenbeck TS. Microbiome control of innate reactivity. Curr Opin Immunol. 2019;56. Elsevier Ltd:107-13.

56. Valles-Colomer M, et al. The neuroactive potential of the human gut microbiota in quality of life and depression. Nat Microbiol. 2019;4(4):623-32 First large-scale, replicated, populationbased study that confirmed associations between gut microbiota and mental health.

57. Malan-Muller S, Valles-Colomer M, Raes J, Lowry CA, Seedat S, Hemmings SMJ. The gut microbiome and mental health: Implications for anxiety- and trauma-related disorders. OMICS J Integr Biol. 2018;22(2) Mary Ann Liebert Inc.:90-107.

58. Li Q, Han Y, Dy ABC, Hagerman RJ. The gut microbiota and autism spectrum disorders. Front Cell Neurosci. 2017;11. Frontiers Media S.A.

59. Dinan TG, Cryan JF. Brain-Gut-Microbiota Axis and Mental Health. Psychosom Med. 2017;79(8) Lippincott Williams and Wilkins:920-6.

60. Benson PR. The impact of child symptom severity on depressed mood among parents of children with ASD: the mediating role of stress proliferation. J Autism Dev Disord. 2006;36(5):685-95.

61. Bruce-Keller AJ, Salbaum JM, Berthoud HR. Harnessing Gut Microbes for Mental Health: Getting From Here to There. Biol Psychiatry. 2018;83(3):214-23 Elsevier USA. Provides an overview of the potential possibilities and current limitations of gut microbiota manipulation to influence mental health.

62. Madison A, Kiecolt-Glaser JK. Stress, depression, diet, and the gut microbiota: human-bacteria interactions at the core of psychoneuroimmunology and nutrition. Curr Opin Behav Sci. 2019;28. Elsevier Ltd:105-10.

63. Bastiaanssen TFS, Cowan CSM, Claesson MJ, Dinan TG, Cryan $\mathrm{JF}$. Making sense of $\cdots$ the microbiome in psychiatry. Int $\mathrm{J}$ Neuropsychopharmacol. 2018;22(1):37-52.

64. Cryan JF, O'Riordan KJ, Sandhu K, Peterson V, Dinan TG. The gut microbiome in neurological disorders. Lancet Neurol. 2020;19(2) Lancet Publishing Group:179-94.

65. Warner BB. The contribution of the gut microbiome to neurodevelopment and neuropsychiatric disorders. Pediatr Res. 2019;85(2) Nature Publishing Group:216-24.

66. Clarke G, Grenham S, Scully P, Fitzgerald P, Moloney RD, Shanahan $\mathrm{F}$, et al. The microbiome-gut-brain axis during early life regulates the hippocampal serotonergic system in a sex-dependent manner. Mol Psychiatry. 2013;18(6):666-73.

67. Möhle L, Mattei D, Heimesaat MM, Bereswill S, Fischer A, Alutis $\mathrm{M}$, et al. Ly6Chi monocytes provide a link between antibioticinduced changes in gut microbiota and adult hippocampal neurogenesis. Cell Rep. 2016;15(9):1945-56.
68. O’Mahony SM, Clarke G, Borre YE, Dinan TG, Cryan JF. Serotonin, tryptophan metabolism and the brain-gut-microbiome axis. Behav Brain Res. 2015;277. Elsevier:32-48.

69. Ogbonnaya ES, Clarke G, Shanahan F, Dinan TG, Cryan JF, O'Leary OF. Adult Hippocampal Neurogenesis Is Regulated by the Microbiome. Biol Psychiatry. 2015;78(4) Elsevier USA:e7-9.

70. Yano JM, Yu K, Donaldson GP, Shastri GG, Ann P, Ma L, et al. Indigenous bacteria from the gut microbiota regulate host serotonin biosynthesis. Cell. 2015;161(2):264-76.

71. Messaoudi M, Lalonde R, Violle N, Javelot H, Desor D, Nejdi A, et al. Assessment of psychotropic-like properties of a probiotic formulation (Lactobacillus helveticus R0052 and Bifidobacterium longum R0175) in rats and human subjects. Br J Nutr. 2011;105(5):755-64.

72. El Aidy M, Kunze S, Bienenstock W, Kleerebezem J. The microbiota and the gut-brain axis: insights from the temporal and spatial mucosal alterations during colonisation of the germfree mouse intestine. Benefic Microbes. 2012;1(3, 4):251-9.

73. Kelly JR, Borre Y, O' Brien C, Patterson E, el Aidy S, Deane J, et al. Transferring the blues: depression-associated gut microbiota induces neurobehavioural changes in the rat. J Psychiatr Res. 2016;82:109-18.

74. Cheung SG, Goldenthal AR, Uhlemann AC, Mann JJ, Miller JM, Sublette ME. Systematic review of gut microbiota and major depression. Front Psychiatry. 2019;10:34, no. FEB. Frontiers Media S.A.

75. Lafuse WP, Gearinger R, Fisher S, Nealer C, Mackos AR, Bailey MT. Exposure to a social stressor induces translocation of commensal lactobacilli to the spleen and priming of the innate immune system. J Immunol. 2017;198(6):2383-93.

76. Bailey MT, "Psychological stress, immunity, and the effects on indigenous microflora.," Adv Exp Med Biol, pp. 225-46, 2016.

77. Dominguez-Andres J, Netea MG. Long-term reprogramming of the innate immune system. J Leukoc Biol. 2019;105(2) John Wiley and Sons Inc.:329-38.

78. Neher JJ, Cunningham C. Priming Microglia for Innate Immune Memory in the Brain. Trends Immunol. 2019;40(4) Elsevier Ltd: 358-74.

79. Skonieczna-Żydecka K, Marlicz W, Misera A, Koulaouzidis A, Łoniewski I. Microbiome-The Missing Link in the Gut-Brain Axis: Focus on Its Role in Gastrointestinal and Mental Health. J Clin Med. 2018;7(12):521.

80. Talbott SM, Talbott JA, Stephens BJ, Oddou MP. Effect of Coordinated Probiotic/Prebiotic/Phytobiotic Supplementation on Microbiome Balance and Psychological Mood State in Healthy Stressed Adults. Funct Foods Heal Dis. 2019;9(4):265.

Publisher's Note Springer Nature remains neutral with regard to jurisdictional claims in published maps and institutional affiliations. 